\title{
Acetaminophen-Induced Hepatotoxicity: a Comprehensive Update
}

\author{
Eric Yoon, Arooj Babar, Moaz Choudhary, Matthew Kutner and Nikolaos Pyrsopoulos* \\ Rutgers New Jersey Medical School, University Hospital, Newark, New Jersey, USA
}

\begin{abstract}
Hepatic injury and subsequent hepatic failure due to both intentional and non-intentional overdose of acetaminophen (APAP) has affected patients for decades, and involves the cornerstone metabolic pathways which take place in the microsomes within hepatocytes. APAP hepatotoxicity remains a global issue; in the United States, in particular, it accounts for more than $50 \%$ of overdose-related acute liver failure and approximately $20 \%$ of the liver transplant cases. The pathophysiology, disease course and management of acute liver failure secondary to APAP toxicity remain to be precisely elucidated, and adverse patient outcomes with increased morbidity and mortality continue to occur. Although APAP hepatotoxicity follows a predictable timeline of hepatic failure, its clinical presentation might vary. $\mathrm{N}$-acetylcysteine (NAC) therapy is considered as the mainstay therapy, but liver transplantation might represent a life-saving procedure for selected patients. Future research focus in this field may benefit from shifting towards obtaining antidotal knowledge at the molecular level, with focus on the underlying molecular signaling pathways.
\end{abstract}

(C) 2016 The Second Affiliated Hospital of Chongqing Medical University. Published by XIA \& HE Publishing Inc. All rights reserved.

\section{Introduction}

Drug-induced liver injury (DILI) is not an uncommon adverse event encountered in clinical practice, since a vast number of

Keywords: Acetaminophen toxicity; Hepatotoxicity; APAP; Paracetamol; Acute liver failure (ALF).

Abbreviations: AIF, apoptosis-inducing factor; ALF, acute liver failure; ALT, alanine aminotransferase; APACHE II, acute physiology and chronic health evaluation II; APAP, acetaminophen; AST, aspartate transaminase; ATP, adenosine triphosphate; DAMP, damage-associated molecular pattern; DILI, drug-induced liver injury; EndoG, endonuclease G; FDA, Food and Drug Administration; GDCA, glycodeoxycolic acid; GDH, glutamate dehydrogenase; GSH, glutathione; HMGB1, high mobility group B1; HPLC, high-pressure liquid chromatography; INR, international normalized ratio; IV, intravenous; JNK, C-Jun-N-terminal kinase; $\mathrm{KCH}$, King's College Hospital; KIM 1, kidney injury molecule 1; MELD, model for end-stage liver disease; miRNA, microRNA; mtDNA, mitochondrial DNA; NAC, $N$-acetyl cysteine; NAPQI, $N$-acetyl-para-benzo-quinone imine; nDNA, nuclear DNA; NK, natural killer; NKT, natural killer T cells; PO, oral; SFN, sulforaphane; SOFA, sequential organ failure assessment; SULT, sulfotransferase; UGT, UDP-glucoronosyl transferase.

Received: 22 December 2015; Revised: 07 April 2016; Accepted: 18 May 2016 DOI: $10.14218 / \mathrm{JCTH} .2015 .00052$.

*Correspondence to: Nikolaos Pyrsopoulos, Division of Gastroenterology \& Hepatology, Rutgers New Jersey Medical School, University Hospital, $185 \mathrm{~S}$. Orange Avenue, Newark, New Jersey 07101, USA. Tel: +1-973-972-5252; Fax: +1-973972-3144, E-mail: pyrsopni@njms.rutgers.edu compounds, including herbs and alternative medications, are metabolized in the liver microsomes. The most detrimental clinical presentation is fulminant liver failure, where patients without a history of liver disease present with hepatic encephalopathy and coagulopathy preceding jaundice. Acetaminophen, also known as APAP (in the United States), paracetamol (in Europe and other areas of the world) or $\mathrm{N}$-acetyl-p-aminophenol, is one of the most commonly utilized compounds worldwide; its use as an anti-pyretic or analgesic drug has been predominant since 1955, particularly due to the fact that it is easily accessible in various formulations as an over-the-counter medication. ${ }^{1}$ Indeed, APAP is reported to be regularly consumed by over 60 million Americans on a weekly basis, making it the most widely utilized analgesic and antipyretic in the United States. ${ }^{2}$ Advertised as safe in doses up to $4000 \mathrm{mg}$ every 24 hours by the United States Food and Drug Administration (FDA), consumption at this dose generally does not yield any toxic effects. ${ }^{2,3}$ Standalone APAP is not the only drug formulation that should be suspected in potential APAP toxicity, however. As such, it may be difficult to recognize APAP toxicity, partly due to its availability in various formulations, such as tablets, liquids, rectal suppositories and intravenous liquids, as well as in combination supplements sold as over-the-counter and prescription products for analgesia. ${ }^{2}$

Reported cases of APAP-induced hepatotoxicity first emerged in the United States in the mid-1980s, and since then all signs point towards a growing incidence. It has been reported that this is one of the most common pharmaceutical products to cause DILI. ${ }^{1-5}$ Mortality rates have been approximated at $0.4 \%$ in overdose patients, translating to 300 deaths annually in the United States. ${ }^{1}$ Although toxic ingestions causing hepatic failure are usually in excess of $150 \mathrm{mg} / \mathrm{kg}$, an increasing number of reports has arisen to suggest that lower doses of APAP may confer acute liver injury and liver failure. ${ }^{1-4}$ A so-called "therapeutic misadventure" phenomenon, as coined by Zimmerman et al., is becoming increasingly acknowledged, as some patients have presented with acute liver failure despite consumption of "safe" doses of APAP. Some of these patients may have some specific risk factors, such as nuances in APAP metabolism at the mitochondrial and molecular level, which are currently under investigation in hopes of further elucidating their contributions to this life-threatening condition.

\section{Epidemiology}

APAP is one of the most commonly used analgesics in the United States and reportedly the most common cause of acute liver failure in the United States. ${ }^{2,3,5}$ In the United States, 
around 30,000 patients are admitted to hospitals every year for treatment of APAP hepatotoxicity. ${ }^{6}$ While there appears to be a near even ratio of patients who take excessive APAP doses knowingly and unknowingly ( $52 \%$ vs. $48 \%$ ), both groups are susceptible to liver failure and generate referrals for liver transplantation. ${ }^{2,6}$ Although the majority of patients experience mild adverse reactions, such as hepatitis, cholestasis or asymptomatic liver enzyme elevation, APAP hepatotoxicity is generally estimated to account for approximately $48 \%$ of acute liver failure diagnoses. ${ }^{1-3}$ Moreover, studies have shown that $29 \%$ of patients with acute liver failure secondary to APAP toxicity undergo liver transplant, and that these cases have a $28 \%$ mortality rate. ${ }^{1}$

With the advent of APAP/opioid combination analgesics and the ever-growing number of prescription narcotics being prescribed by medical practitioners, a larger number of patients have been placed in harm's way as they are at significant risk of APAP hepatotoxicity. Less than a decade ago, in 2010, over 130 million prescriptions for APAP/hydrocodone were filled in the United States. ${ }^{3}$ Reportedly, $63 \%$ of unintentional overdoses of APAP occur with use of the opioid/ APAP combination, with an additional $17 \%$ of adults suffering liver injury. ${ }^{5,6}$ Fortunately, the United States FDA has recognized the dangers of the prescription combination of APAP and narcotic analgesics and, in January 14, 2014, implemented a fixed amount of APAP approved per dosage unit of combination analgesics tablets in effective practice. Epidemiologic studies have demonstrated that at least $6 \%$ of filled prescriptions for either acetaminophen alone or acetaminophen in combination with opioids exceeded $4000 \mathrm{mg}$ per day of acetaminophen, which is a cause for real concern. ${ }^{3,5,6}$ Further epidemiologic studies have demonstrated that there is a true lack of knowledge regarding the harmful potential of APAP. It is not uncommon for patients to confuse non-steroidal antiinflammatory (NSAIDs) medications, such as naproxen or ibuprofen, with APAP. While providing health literacy education to teach distinguishment of APAP and NSAIDs is important, some have thought it possibly more beneficial to mitigate the chance of inappropriate APAP over-ingestion by reducing the pack size of APAP preparations sold, which they believe may reduce the incidence and severity of APAP hepatotoxicity. ${ }^{7}$ What is clear is that APAP overdose and hepatotoxicity is a preventable public health concern that deserves awareness by both clinicians and the patient population alike.

\section{Pharmacology and Hepatotoxicity}

APAP absorption occurs rapidly in the duodenum, owing to its property as a weak acid. ${ }^{8}$ If a patient consumes food around the same time of APAP ingestion, there may be a delay in the time of, but not the extent of, drug absorption. ${ }^{8}$ Much like concurrent food consumption causing time-delay in APAP absorption, a patient with chronic liver disease is at risk of prolonged drug serum half-life (by an average of 2.0 to 2.5 hours, and up to more than 4 hours), especially if extended-release APAP formulations are consumed. While an overdose of APAP yields peak serum concentrations $(10-20 \mu \mathrm{g} / \mathrm{mL})$ within 4 hours, a patient taking the medication safely will achieve peak concentrations within 1.5 hours, with a half-life of $1.5-3$ hours. ${ }^{8}$

APAP metabolism occurs within liver microsomes at the microscopic level. While the pathways are well elucidated and the fine details of their molecular mechanisms are beyond the scope of this manuscript, it is worth noting that not all patients suffer the same fate when it comes to APAP ingestion and hepatotoxicity. There are three phases of APAP metabolism. The majority ( $\sim 90 \%)$ of the APAP is funneled into phase II metabolic pathways, in which APAP conjugation is catalyzed by UDP-glucuronosyl transferases (UGT) and sulfotransferase (SULT), with conversion to glucouronidated and sulfated metabolites that are eliminated from the body in the urine ${ }^{8}$ (Figure 1). A small, measurable amount of APAP $(\sim 2 \%)$ is excreted in the urine without having undergone any metabolism. ${ }^{8}$ Another portion of APAP $(\sim 10 \%)$ is shunted by hepatic cytochrome CYP 2E1 (to a lesser extent with CYP 1A2 and 3A4) to phase I oxidation, in which a highly reactive toxic metabolite, $\mathrm{N}$-acetyl-para-benzo-quinone imine (NAPQI), is formed. ${ }^{9-13}$ Phase III involves metabolite transport in the form of biliary excretion that requires transporters. ${ }^{8}$

APAP hepatotoxicity occurs through formation of the noxious NAPQI metabolite, which is present in excessive quantities, as augmented by features of glutathione (GSH) depletion, oxidative stress and mitochondrial dysfunction leading to depletion in adenosine triphosphate (ATP) stores. ${ }^{3,9,13}$ There is evidence to support the theory that the metabolic activation of APAP generates NAPQI that binds to a number of cellular proteins, especially mitochondrial proteins. Adherence to mitochondrial proteins, especially in the setting of GSH depletion, is important because mitochondrial protein binding depletes native antioxidant functions and also alters the mitochondrial ATP-synthase $\alpha$-subunit, leading to ineffective ATP production. ${ }^{9,13}$

Other mechanisms of hepatotoxicity include the formation of toxic free radicals, such as peroxynitrite, from the reaction of superoxide and nitric oxide, subsequently forming nitrotyrosine adducts inside the mitochondria. ${ }^{9,13} \mathrm{GSH}$ repletion not only provides surplus cysteine as an energy substrate for the Krebs cycle, it also serves the important role of scavenging for free radicals and peroxynitrite. ${ }^{9,10}$ Mitochondria, which are critical for cellular respiration and metabolism, suffer damage to their own mitochondrial DNA by the actions of reactive oxygen species and peroxynitrite compounds, and they have been directly implicated in the process leading to cessation of ATP synthesis. ${ }^{9,11}$

Many biochemical studies have been performed in murine models. However, when HepaRG stem-cell derived human hepatocytes were subjected to APAP, the same mechanisms of hepatotoxicity were demonstrated-starting with GSH depletion and moving through protein adduct formation, superoxide and peroxynitrite formation and lysosomal iron uptake into mitochondria. ${ }^{9-11,13,14}$ Oxidative stress compounded with lysosomal iron uptake within the mitochondria leads to mitochondrial membrane dysfunction via disruption of the mitochondrial membrane permeability transition pore, triggering cell necrosis. ${ }^{9-11,13,14}$ Organelle swelling leads to cellular necrosis and the release of mitochondrial contents, such as apoptosis-inducing factor (AIF) and endonuclease G (EndoG), which in turn migrate to the nuclei and cause DNA fragmentation. ${ }^{12}$ Cellular swelling, karyolysis, karyorrhexis, vacuolization, inflammation and release of cellular contents (alanine aminotransferase, ALT) are key processes of hepatocyte necrosis and related death in humans, as shown by the biochemical evidence of severe elevations in aminotransferases, especially ALT. ${ }^{9-11}$

In non-toxic ingestion of APAP, the processing of NAPQI occurs with rapid conjugation by hepatic GSH to form nontoxic mercaptate and cysteine compounds that are excreted in urine. ${ }^{8}$ The body requires a delicate balance between production and detoxification/transport of harmful reactive species and protein adducts, and when these defenses are 
Yoon E. et al: Acetaminophen-induced liver toxicity

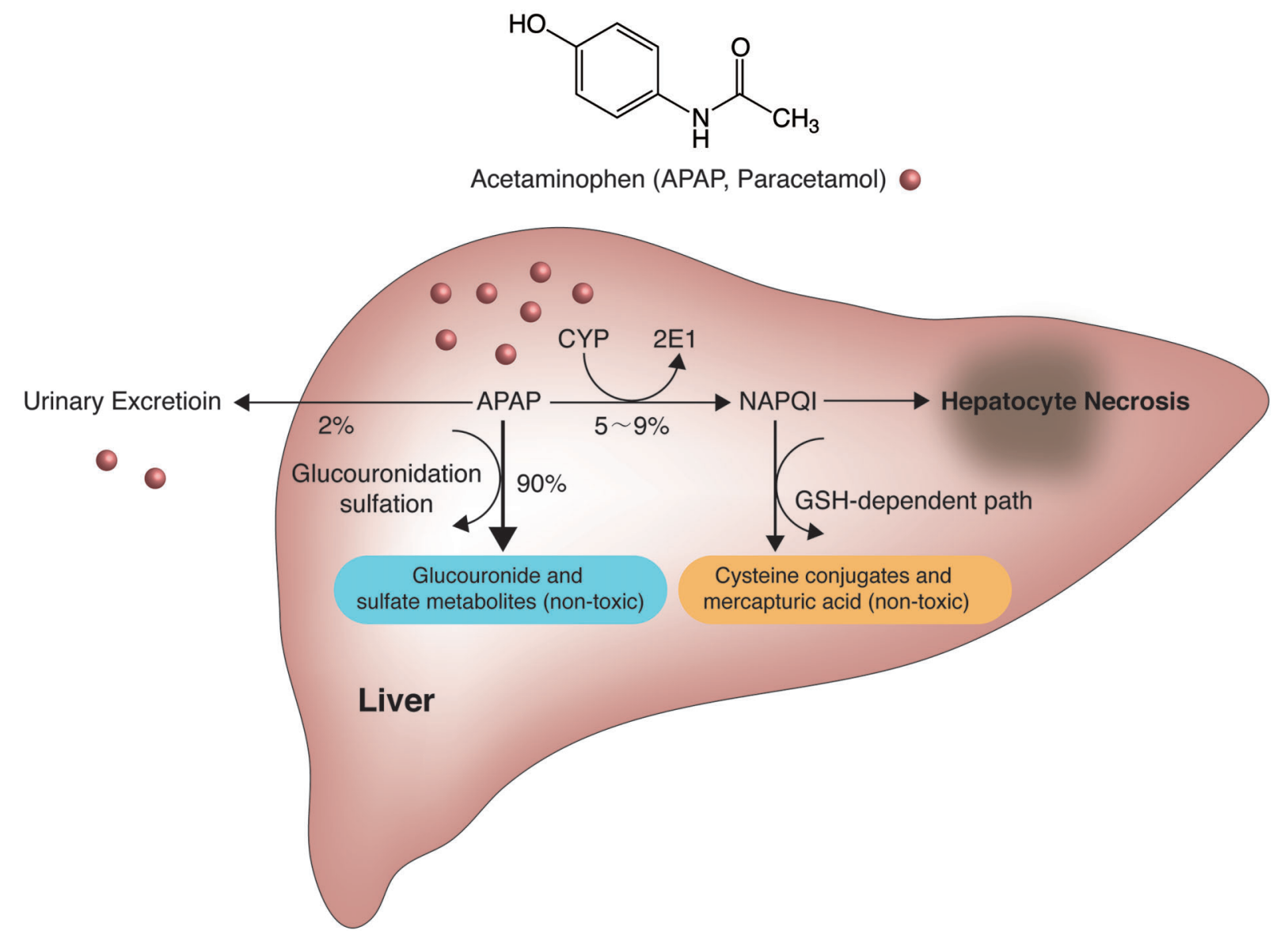

Fig. 1. Acetaminophen (APAP) metabolic pathway.

overwhelmed at hepatotoxic doses of APAP, the glucoronidation and sulfonation pathways become saturated, pushing the majority of APAP to be metabolized to NAPQI by the CYP 2E1 pathway and resulting in GSH depletion and build-up of toxic NAPQI. ${ }^{9,10}$ Myeloperoxidase and cyclooxygenase-1 are enzymes that also function in the processing of NAPQI into non-reactive metabolites. In addition, hepatocytes have the ability to induce innate and adaptive immune defenses to dampen the inflammatory response during necrosis. ${ }^{11}$ It has been suggested that perhaps the role of the innate immune system, as mediated by the natural killer (NK) and natural killer T cells (NKT) which are abundant in hepatocytes, is to cause the release of pro-inflammatory cytokines and chemokines that enhance hepatocellular cytotoxicity. ${ }^{15}$ Damageassociated molecular pattern (DAMP) species released into the circulation, such as nuclear fragments and mitochondrial DNA (mtDNA), appear to recruit inflammatory cells via the innate immune system as the liver's attempt to remove necrotic cell debris and promote the liver's recovery phase. ${ }^{12,16}$

APAP-induced cell death of hepatocytes takes on the characteristic changes of necrosis. The hepatotoxicity of APAP rests predominantly with the highly toxic and reactive compound NAPQI, which forms covalent bonds with sulfhydryl groups on cysteine and lysine molecules within the mitochondria of hepatocytes and which spontaneously reacts with GSH and binds to hepatic proteins. ${ }^{8,11}$ The protein adducts formed, specifically those of mitochondrial protein adducts, lead to oxidative injury and hepatocellular necrosis. An approximately $70 \%$ reduction of GSH has been proposed as the threshold for protein adduct binding to occur; however, this theory has been called into question as APAP-protein adducts have been detected at 1 hour after APAP treatment, a time that that would precede GSH depletion. ${ }^{8}$ It is important to note, however, that a clear inverse relationship has been demonstrated between GSH concentration and APAP metabolic activation activity. ${ }^{8}$ Findings from current studies of APAP hepatotoxicity seem to favor the mechanism of toxic injury being related to mitochondrial and endoplasmic reticula leading to hepatocyte injury and necrosis.

\section{Factors influencing APAP-related hepatotoxicity}

The most essential determining factor in both the development and severity of APAP hepatotoxicity is the drug's ingested dose, but some argue that the length of time from APAP ingestion to $N$-acetylcysteine (NAC) therapy ("time to NAC") is equally if not more important. ${ }^{15,17-20}$ Many other factors are confluent in the development and severity of liver injury after APAP over-ingestion (Table 1). Liver metabolism during glucouronidation or sulfation, CYP activity and maintenance of hepatic GSH supply depends on patient factors such as age, nutritional status, pre-existing liver disease, concurrent use of alcohol and other liver-metabolized medications, genetic 
Table 1. Factors influencing APAP-related hepatotoxicity

\begin{tabular}{|c|c|}
\hline Factors & Clinical implications \\
\hline $\begin{array}{l}\text { Dose and } \\
\text { pattern of use }\end{array}$ & $\uparrow$ APAP toxicity with acute, high doses \\
\hline \multirow[t]{2}{*}{ EtOH } & $\begin{array}{l}\downarrow \text { APAP toxicity with acute EtOH } \\
\text { ingestion }\end{array}$ \\
\hline & $\begin{array}{l}\uparrow \text { APAP toxicity with chronic EtOH } \\
\text { ingestion }\end{array}$ \\
\hline $\begin{array}{l}\text { Herbs and } \\
\text { medications }\end{array}$ & $\begin{array}{l}\uparrow \text { APAP toxicity with impedance of } \\
\text { hepatic glucouronidation }\end{array}$ \\
\hline $\begin{array}{l}\text { Age and genetic } \\
\text { factors }\end{array}$ & $\begin{array}{l}\uparrow \text { APAP toxicity with advancing age and } \\
\text { impaired glucouronidation }\end{array}$ \\
\hline $\begin{array}{l}\text { Nutritional } \\
\text { status }\end{array}$ & $\uparrow$ APAP toxicity in malnourished patients \\
\hline $\begin{array}{l}\text { Chronic liver } \\
\text { disease }\end{array}$ & $\begin{array}{l}\uparrow \text { APAP toxicity, especially in chronic } \\
\text { EtOH abuse }\end{array}$ \\
\hline
\end{tabular}

Abbreviations: APAP, acetaminophen; EtOH, ethyl alcohol.

predispositions, and most importantly, the acuity or chronicity of APAP overuse. $3,15,17,20-30$

\section{Dose and pattern of use}

Zimmerman's "therapeutic misadventure" describes acute liver injury following APAP consumption and failure of APAP therapy, when used at or below the daily maximum recommended dose of $4000 \mathrm{mg}$. However, there are many other patients who intentionally over-ingest APAP (with clear evidence from careful history taking and measured APAP levels), or the more indolent and accidental over-ingestion of doses over $4000 \mathrm{mg}$ per day (not as obvious when eliciting the patient history), which we would like to term "therapeutic misfortune." The United States FDA clearly states "severe liver damage may occur if you take more than $4000 \mathrm{mg}$ of acetaminophen in 24 hours." ${ }^{31}$ In these extreme circumstances, prompt medical assessment and therapeutic intervention is necessary to administer therapy that will allow for salvage of a damaged and/or failing liver.

There is no question that the upper threshold on the maximum daily allowed dose of APAP has been the subject of controversy. An example of such is the voluntary adjustments made by McNeil, the pharmaceutical producer of the Tylenol $^{\circledR}$ brand of APAP, wherein the maximum daily APAP dose was reset from $4000 \mathrm{mg}$ to either $3000 \mathrm{mg}$ per day or $3250 \mathrm{mg}$ per day for consumers taking the $500 \mathrm{mg}$ or $325 \mathrm{mg}$ tablet preparations, respectively. ${ }^{32}$ Many of the other manufacturers have not followed suit, however. For increased patient safety in APAP consumption, the FDA has worked to mitigate APAP hepatotoxicity by legislating regulations to limit prescription combination drug products to have no more than $325 \mathrm{mg}$ of APAP per dosage unit and have urged healthcare providers to cease prescribing combination drug products with more than $325 \mathrm{mg}$ of APAP per tablet. ${ }^{31}$

One study reported that ingestion of the recommended maximum amount of $4000 \mathrm{mg}$ of APAP for 2 weeks resulted in the asymptomatic elevation of alanine transaminase, up to three times normal, in $40 \%$ of patients. ${ }^{19}$ These transaminase elevations did not amount to any clinical significance, and after the APAP was discontinued the transaminase levels returned to normal. ${ }^{19}$ Yet, while asymptomatic, chronically elevated aminotransferases may be of concern to healthcare providers, leading to further costly diagnostic studies or changes/restrictions in necessary medications.

Alcohol

The pairing of alcohol and APAP is a reality that deserves attention, as both substances are ubiquitous in society. Furthermore, individuals with the intent of self-harm may over-ingest both substances. Intuitively, the co-ingestion of alcohol and APAP is expected to cause acute hepatotoxicity. As it turns out, the manner in which alcohol is consumed plays a significant role in who may or may not suffer APAP-related hepatotoxicity. ${ }^{8,11,20-24}$ The interplay between alcohol and APAP, which is a competitive substrate for CYP 2E1 metabolism, is complex. ${ }^{20-24}$ According to a prospective observational study of approximately 360 patients who presented with acute APAP overdose, the concurrent acute ingestion of alcohol and APAP resulted in the prevalence of hepatotoxicity, as defined by ALT > $1000 \mathrm{U} / \mathrm{L}$ or an international normalized ratio (INR) of $>1.3$, in $5.1 \%$ of patients versus $15.2 \%$ of patients who did not consume alcohol among all of the APAP consumers who presented as above or below the "200-line" on the Rumack-Matthew nomogram; moreover, this finding was repeated regardless of the time between ingestion and assessment. ${ }^{21}$ Thus, acute ethanol ingestion may serve as a protective mechanism against APAP hepatotoxicity, but whether this phenomena takes place only in alcoholic patients (versus nonalcoholic patients) is unclear. ${ }^{8,20-22}$ The mechanism behind this interesting finding appears to be ethanol's competitive utilization of the CYP 2E1 substrate, which diminishes the NAPQI byproduct of phase I APAP metabolism. ${ }^{21,22}$ Another suggested hypothesis is that ethanol may directly enhance the activity of $\mathrm{NAD}(\mathrm{P}) \mathrm{H}$ :quinone reductase, serving to reconvert quinone metabolites back to native APAP and thereby limiting the accumulation of toxic metabolites. ${ }^{21}$

Chronic alcohol ingestion, however, augments APAP hepatotoxicity by up-regulating, enhancing and increasing the synthesis and activity of CYP 2E1 and decreasing GSH stores and synthesis, thus enhancing liver necrosis and worsening prognosis. ${ }^{5,8,21}$ Chronic alcoholism and APAP overdose may potentiate liver failure, but there is no indication that the combination of alcoholism and taking therapeutic amounts of APAP will necessarily cause hepatotoxicity. ${ }^{21}$ Therefore, one has to be wary of characterizing all alcoholics as being at higher risk of APAP hepatotoxicity. Regular ethanol consumption of greater than $250 \mathrm{mg} / \mathrm{dL}$ appears to be the threshold for enhancement of CYP 2E1 enzymatic activity; this condition represents individuals who drink 18 or more standard alcoholic drinks. ${ }^{21} \mathrm{~A}$ more reasonable explanation of why chronic alcohol abusers have higher APAP-related hepatotoxicity is a tendency among these individuals to delay seeking medical attention after APAP ingestion, which would prolong their "time to NAC"-the only demonstrated treatment available for salvage of a hepatotoxic liver.

The data suggests that chronic alcohol consumers who experience "therapeutic misadventure" are at increased risk of APAP hepatotoxicity. ${ }^{20-24}$ It appears that a single dose of APAP, at $325 \mathrm{mg}$ to $500 \mathrm{mg}$ for as-needed analgesic or anti-pyretic purposes, will not lead to acute APAP hepatotoxicity in an alcoholic patient, especially if APAP is taken less than 8 hours from alcohol consumption. APAP is often prescribed, 
and can be prescribed, to patients who require an anti-pyretic or analgesic despite recent history of alcohol ingestion.

\section{Herbals and medications other than APAP}

There are many prescribed and over-the-counter substances that interact with liver metabolism; these interactions can result in harmful or protective tendencies, and have been reported for a plethora of particular herbs and medications. Medications that stimulate the CYP system can predispose patients to APAP hepatotoxicity by causing enhanced production of NAPQI via the oxidative pathway. ${ }^{25}$ Case series reports have concluded that in $50 \%$ of adult patients with APAPinduced acute liver failure, the life-threatening condition was preceded by ingestion of potentially hepatotoxic herbs within 8 weeks of hospital presentation. ${ }^{4}$ The prescription agents known for their hepatotoxic tendencies include anti-epileptic drugs, such as phenobarbital, phenytoin and carbamazepine, as well as anti-tuberculosis drugs, such as isoniazid and rifampin. Over-the-counter herbs and dietary supplements, such as St. John's wort, garlic and germander, may mechanistically enhance the CYP system. ${ }^{1}$ An alternative mechanism by which medications may enhance APAP hepatotoxicity involves competitive utilization of the glucouronidation pathway, resulting in increased metabolism towards the CYP system and thus enhanced formation of hepatotoxic substrates. ${ }^{25}$ Importantly, opioid therapies can reduce the level of hepatic GSH and thereby potentiate APAP-induced liver injury. ${ }^{8}$

It is important to note that some natural products and remedies have been demonstrated to have protective potential against APAP hepatotoxicity. ${ }^{8}$ One proposed mechanism underlying this phenomenon is the competitive utilization of CYP 2E1 in herbal drug metabolism that would decrease APAP's conversion into its toxic metabolites, such as NAPQI. ${ }^{25,33,34}$ The Schisandra plant species, ubiquitous in its use as a traditional East Asian herb, has been used in Korea, China and Japan as a remedy for hepatitis; in addition, the Wuzhi Tablet, a recently introduced alcohol extract preparation of S. sphenanthera, has shown significant hepatoprotective effects against APAP-induced liver injury. ${ }^{34}$ Treatment with one particular herbal agent, Schisandrol B, the active extractant from S. sphenanthera, has been shown to decrease APAP hepatotoxicity as well as to lead to features of liver regeneration by inhibiting NAPQI-initiated injury via the blockade of CYP activity, the down-regulation of p53/p21 and the up-regulation of other signaling pathways to facilitate hepatic tissue repair. ${ }^{34}$

\section{Nutritional status}

The collective evidence shows a clear association between malnourishment and APAP hepatotoxicity. Malnutrition is associated with conditions consistent with GSH depletion, which may support its role of placing patients at further risk for hepatotoxicity. In a poor nutritional state, GSH reserves become easily exhausted and hepatic glucouronidation is stunted, leading to decreased levels of available GSH that would otherwise be scavenged for formation of reactive metabolites in phase I of APAP metabolism. Poor nutritional status is often associated with chronic alcoholism, and thus alcoholic patients with APAP-induced hepatotoxicity may fall victim to both decreased body GSH stores as well as enhancement of the CYP enzymatic activity. ${ }^{30}$
Even though chronic malnutrition is common among liver disease patients, there is a subset of malnourished patients, namely those with the psychiatric illness anorexia nervosa, which merits special attention. This subpopulation may undertake intentional APAP overdoses, representing intent of self-injury. Patients with anorexia nervosa have low GSH stores already, as is expected in the malnourished state, but their rate of CYP 2E1 activity is also diminished; thus, the risk of APAP toxicity appears not to be exacerbated in these nutritionally-depleted patients in particular, due to this unique circumstance. ${ }^{29}$

\section{Phosphate in APAP hepatotoxicity}

Early murine models demonstrated that mice with adequate ATP supply underwent hepatocyte apoptosis when exposed to excessive APAP. In fasting animals, the opposite was true, with necrosis being the more prominent response to excessive APAP. Phosphate has historically been associated with poor outcomes in patients with APAP hepatotoxicity, with early studies showing an association between low phosphate levels and increased morbidity and mortality. ${ }^{35}$ However, findings from more recent studies have led to the proposal that patients with APAP hepatotoxicity and high levels of serum phosphate have worse outcomes. Levels greater than $3.7 \mathrm{mg} / \mathrm{dL}$ at $48-72$ hours post-ingestion have been reported as associated with higher mortality from acute liver failure. ${ }^{36,37}$ Findings from other research studies have contradicted this proposition, however, showing that the association between hyperphosphatemia and liver failure is only evident in patients with acute renal failure (with $50-80 \%$ of patients with acute liver failure having concomitant acute renal failure ${ }^{38}$ ). It is wellknown that with acute kidney injury, serum phosphate levels rise; disruption of pathways necessary for adequate cell regeneration and ATP production facilitates serum phosphate levels remaining high, with some researchers postulating an association of this with increased mortality. The association between hypophosphatemia and better clinical outcomes seems to be secondary to enhanced cell regeneration with hepatocyte phosphate uptake from the serum to generate ATP. ${ }^{39,40}$ Given the importance of serum phosphate levels, members of the Acute Liver Failure Study Group (commonly referred to as ASFLG) have included serum phosphate levels in their prognostic scoring system for mortality related to APAP hepatotoxicity. ${ }^{41}$

\section{Age and genetic factors}

APAP, an analgesic utilized by patients from infancy into the geriatric years, shows age-related hepatotoxic tendencies. In general, younger patients are better able to overcome acute liver failure as a result of APAP hepatotoxicity, probably due to the larger hepatic cell mass that is present in this population before the cell damage occurs, as well as the better capacity of those cells for non-toxic metabolism and their improved capacity for regeneration. ${ }^{20}$ Patients over 40 years old who overdose on APAP portend a higher risk of acute liver failure, liver transplantation and death. ${ }^{5}$ In general, APAP metabolism appears age-dependent, with elderly patients being at higher risk of hepatotoxicity after acute overdose of APAP than the pediatric population. ${ }^{42}$ Considering the current widespread use of APAP alone and in opiate combinations among adults of advanced age (to treat cancer pain or chronic musculoskeletal pains), one must not forget 
to monitor patients for complications of long-term and highdose APAP use.

Various genetic polymorphisms in CYP enzymes and their levels of activity may explain why APAP is metabolized with excessive or diminished oxidative capabilities. ${ }^{26,43,44}$ The enzymes UGT (glucouronidation), SULT, CYP 450, GST, $\mathrm{N}$-deacetylase (deacetylation), NAT2 (deacetylation), and fatty acid amide hydrolase are involved in APAP metabolism and have been shown to be related to both hepatic and nephrotoxic effects of the analgesic medication. ${ }^{45}$ It appears that genotypic changes of these enzymes leads to potentially different risk/benefit ratios when APAP is ingested. Comparative studies of Caucasian and African populations showed that more mercapturic acid and cysteine conjugate metabolites were recovered in the former (at nearly a 2:1 ratio), indicating that increased hepatic metabolism occurs in that group. ${ }^{43}$ Another study, which compared Caucasian and Chinese populations indicated the increased susceptibility of the former to APAP hepatotoxicity reflects the lower CYP 2E1 and 2D6 activity in the latter. ${ }^{45}$ Thus, although no specific limitations on APAP intake have been proposed based on ethnic background, further studies on this topic may yield useful clinical information about the safety profile amongst a diverse patient population.

\section{Chronic liver disease}

APAP metabolism is reduced in patients with cirrhotic livers, as compared to those with normal (non-diseased) livers. Chronic liver disease patients who use alcohol infrequently do not appear to be at an elevated risk of developing APAP hepatotoxicity. ${ }^{21,22,24}$ Although ideally a different choice of anti-pyretic or analgesic may be used, a less than $4000 \mathrm{mg}$ per day use of APAP in the cirrhotic patient may be safe in the short term. More conservative dose limits, such as $2000 \mathrm{mg}$ per day, have been recommended, especially for patients in whom the liver disease is marked with hepatic decompensation or active alcohol abuse. ${ }^{21}$

\section{Pregnancy}

APAP is considered the most frequently prescribed analgesic in pregnancy. ${ }^{46}$ Pregnancy is a high-risk state for many medications, and APAP is no exception. Unfortunately, APAP toxicity in pregnancy can result in significant morbidity and mortality for both the mother and the fetus. ${ }^{46}$ APAP can freely cross the selective maternal-fetal barrier of the placenta, after which it can then be metabolized by fetal hepatocytes, causing fetal hepatic necrosis if appropriate therapy with NAC is not administered in a timely manner. ${ }^{47}$ Even though there is heightened activity of glucouronidation and oxidative pathways, causing the increased clearance of APAP in pregnancy, no evidence exists to suggest pregnancy as a predisposing risk factor for APAP toxicity. ${ }^{1}$ Moreover, despite that fact that APAP can cross the placental barrier, the only instances when the fetus has been shown to be at risk is when the mother suffers from severe APAP hepatotoxicity, which is usually apparent based on the history of presenting illness. Some case reports have described loss of pregnancy following high-dose ingestion of APAP (up to $29 \mathrm{~g}$ ) within a day of hospital presentation and others have described successful fetus delivery following ingestion of $15 \mathrm{~g}$ and aggressive NAC rescue therapy. ${ }^{48}$ Should either APAP "therapeutic misadventure" or intentional overdose be suspected, aggressive antidote intervention with
NAC should be of the utmost priority since the benefit of therapy may outweigh the risks of untreated APAP toxicity. ${ }^{1,47}$ Although more studies retrospectively investigating pregnant women would be helpful, for now the judicious use of APAP should be advised as APAP remains the most common drug overdose in pregnancy. ${ }^{46}$

\section{Clinical manifestations}

Identification of APAP overdose is critical, as significant morbidity and mortality may be prevented with early therapy. Many patients have only minimal and non-specific symptoms that are comparable to viral prodrome; these symptoms include malaise, nausea with or without vomiting, and abdominal pains. There are four established sequential stages of APAP hepatotoxicity, which should be considered upon presentation to clinic. It is vital, however, to keep in mind that while each stage is designated by a general time range following the APAP over-ingestion, clinical symptoms and laboratory results are dependent on the formulation (combined opiate-APAP preparations, extended-release, etc.) or doses of APAP ingested, including co-ingestions (i.e. chronic or acute alcohol ingestion, herbal supplements, or prescription drugs, as previously discussed), and the existence of preceding liver disease.

Stage $I$ occurs within the first 24 hours of ingestion and is characterized by the non-specific symptoms of nausea, vomiting, malaise, lethargy and diaphoresis. Aspartate transaminase (AST) and ALT values are usually normal, although in immense overdose, elevated values can be detected in as little as 8-12 hours.

Stage II occurs within 24 to 72 hours and is characterized by improvement or resolution of stage $I$ symptoms (also known as the latent period). However, elevations of AST and ALT typically begin to occur. Severe cases of APAP overdose can present with tender hepatomegaly (with right-upper quadrant pain), jaundice and coagulopathy. Approximately $1 \sim 2 \%$ of patients may also experience renal failure in the setting of acute tubular necrosis with or without hepatic necrosis. ${ }^{48}$

Stage III occurs within 72 to 96 hours after the initial APAP overdose, and is noted by the return of stage $I$ symptoms along with marked AST and ALT elevations (possibly > 3000 IU/L) in conjunction with jaundice, encephalopathy, coagulopathy and lactic acidosis. Maximal liver injury occurs in this stage. Renal failure, and on rare occasions pancreatitis, can occur as a complication. ${ }^{49}$ This stage has the highest risk of mortality, which is most frequently due to multi-organ failure. Lactic acidosis portends a poor prognosis (by King's College Hospital ( $\mathrm{KCH}$ ) criteria), and the mechanism of lactic acidemia is two-fold: NAPQI being present in excess and causing mitochondrial dysregulation, followed in subsequent hours by tissue hypoxia and decreased hepatic metabolism and clearance of lactate, leading to a "two-hit" effect on worsening of the lactic acidosis condition. ${ }^{12,13}$ Certain objective data, such as a prothrombin time peaking at > 180 seconds, along with prothrombin time that continues to rise beyond 4 seconds after the APAP overdose, confers about a $90 \%$ mortality without liver transplantation. ${ }^{50}$ If a patient becomes obtunded and comatose as a result of the APAP overdose, intubation with mechanical ventilation becomes necessary.

Stage IV occurs after 96 hours following the recovery from stage III. Normally, stage IV lasts between 1-2 weeks, but its duration can be prolonged depending upon the severity of the ingestion as well as the preparation of APAP ingested. 
If liver biopsies had been performed in previous stages, the histologic recovery period may take several months longer than the clinical recovery. Chronic hepatitis has not been reported as a complication of APAP overdose-associated acute liver failure. ${ }^{1}$

Poor prognostic signs include multi-organ failure, which may involve cerebral edema, renal failure, profound hypoglycemia and lactic acidosis, any signs of which should prompt an immediate liver transplant evaluation. ${ }^{4,6,8,50,52,53}$ In clinical practice, signs of unintentional toxic ingestion typically present later, when the above-mentioned complications are already apparent and have progressed. Indeed, further studies to elucidate the prevalence of APAP-related hepatotoxic patients may be beneficial to identify the clinical stage of APAP hepatotoxicity in order to better triage patient treatment, improve disposition, and design the best follow-up.

APAP hepatotoxicity is one of few bona fide causes of liver injury that can raise serum aminotransferases $>10,000 \mathrm{IU} / \mathrm{L}$. The most worrisome time for maximal organ damage occurs between 3 and 5 days following the acute ingestion, straddling stage III and stage IV hepatotoxicity; thus, prompt recognition of APAP toxic ingestion with expedient initiation of therapeutic intervention becomes important to prevent acute liver failure.

\section{General approach and diagnostic tools}

Patient history and physical examination are paramount, especially in delineating the time course and formulation of APAP ingested. APAP level measurements are commonly performed when a patient initially presents in the acute care setting with a toxic ingestion of unknown substance, altered mental status and/or suspicion of intent of self-harm. It is critical to obtain a 4-hour APAP level, or as close to a 4-hour level as possible, as this value guides therapy and impacts patient outcome. Upon presentation, additional laboratory studies are necessary to obtain other important clinical parameters, including arterial blood gas (to investigate acid/ base status), coagulation profile, basic metabolic panel, hepatic function tests, and urine drug screen (to determine possible co-ingestions). ${ }^{54} \mathrm{~A}$ negative APAP reading should not be interpreted as APAP-related hepatic injury having not taken place, as levels may be undetectable while liver damage has already occurred. However, clinicians should be cautious in interpreting APAP levels in patients with direct hyperbilirubinemia $>10 \mathrm{mg} / \mathrm{dL}$, as a falsely elevated APAP concentration can be the result of bilirubin's interaction with the enzymatic measurement of APAP. ${ }^{55}$ This clinical context becomes especially important because DILI can manifest significant hyperbilirubinemia.

\section{Evaluations after acute single overdose}

Use of the Rumack-Matthew nomogram in management of APAP overdose is acceptable when the acuity of ingestion is known to be within 24 hours. ${ }^{54,56-59}$ The nomogram plots the independent time in hours versus APAP concentration. The important values from the nomogram plotting are points on the "probable toxicity line," which include an APAP level of $200 \mu \mathrm{g} / \mathrm{mL}$ at 4 hours and $25 \mu \mathrm{g} / \mathrm{mL}$ at 16 hours after acute ingestion. Patients who fall above these levels at the appointed times are at risk of severe hepatotoxicity (defined as AST > $1000 \mathrm{IU} / \mathrm{L}$ ). ${ }^{56-62}$ NAC therapy is recommended in such clinical scenarios, to overcome the reported incidence rates of $60 \%$ for severe hepatotoxicity and $5 \%$ for mortality. ${ }^{57}$ A "high toxicity line" also exists parallel to the "probable toxicity line", and this begins at $300 \mu \mathrm{g} / \mathrm{mL}$ at 4 hours, equating to a $90 \%$ incidence of severe hepatotoxicity and $24 \%$ mortality. ${ }^{57}$ In the United States, Australia and New Zealand, a more conservative measurement has been established, starting at a 4-hour APAP concentration of $150 \mu \mathrm{g} / \mathrm{mL}$ and known as the "treatment line," which is intentionally set lower to account for inaccuracies in the history of APAP ingestion and inherent laboratory error in APAP measurement. ${ }^{54,59}$ This line sits $25 \%$ below the "probable toxicity line." Studies have already shown that the "treatment line" (also known as the "150 line") serves well to identify and help protect high-risk patients who may already have diminishing APAP levels. ${ }^{54,59}$ Further conservative measures to decrease the APAP measurement threshold to a "100 line" have not presented demonstrated increases in patient health success rate; rather, use of such a strategy would subject more patients to overtreatment without much added benefit, leading to increasing healthcare costs as patients are often treated for acute APAP hepatotoxicity in medical intensive care units to ensure close monitoring of vital signs and the ability for frequent neurologic assessments. ${ }^{54,59}$

In general, due to the benign nature of NAC therapy, if there is any doubt about the concentration of serum APAP or the timing of ingestion, treatment with NAC is safe and indicated. The initial purpose of the Rumack-Matthew nomogram was for acute ingestions of APAP and its use has not been validated for chronic ingestions, extended-release preparations or co-ingestions of APAP with other substances that may be hepatically cleared. This limitation proves clinically significant as many individuals with APAP over-ingestion encompasses this broad-spectrum population. ${ }^{21}$

\section{Evaluations after repeated overdoses}

Patients with unintentional APAP overdoses have usually ingested APAP often over many days as an analgesic or antipyretic therapy. Symptoms of hepatotoxicity may have already begun by presentation. Jaundice, right-upper quadrant pain, nausea, vomiting, hepatomegaly and encephalopathy indicate high levels of APAP ingestion, and thus when these symptoms are observed the patient's APAP level should be checked. ${ }^{27}$ The Rumack-Matthew nomogram that was previously described is not applicable in this case, and instead treatment with NAC would be appropriate for a finding of APAP levels $>20 \mu \mathrm{g} / \mathrm{mL}$, with or without ALT elevation. ${ }^{56,60}$ NAC should also be administered when a patient has excessive APAP intake with elevated ALT levels, even though serum APAP levels are undetectable. NAC does not appear to be of benefit when APAP levels are undetectable, patients are asymptomatic, or ALT level is normal.

\section{Evaluations after established hepatotoxicity and liver failure}

Because the overwhelming majority ( $90 \%)$ of patients with APAP hepatotoxicity will recover spontaneously from their acute ingestion and instead require appropriate therapy, with intensive care monitoring using cardiac telemetry and frequent laboratory checks. Admission at a liver transplant facility is important for patients who develop signs of acute liver failure, which include encephalopathy, coagulopathy and metabolic acidosis. The workup and management of fulminant hepatic failure does not differ under conditions of APAP 
hepatotoxicity, except for the use of NAC as an antidote. Although the APAP toxicity-associated acute liver failure has better outcome than other causes of liver failure, mortality is still significant in $\sim 30 \%$ of cases without a liver transplant (for those who are deemed in need of a transplant). ${ }^{51,52}$

The development of a prognostic model for survivability of APAP hepatotoxicity, with or without liver transplant, was an important achievement to delineate need for emergency transplantation. The $\mathrm{KCH}$ in London, United Kingdom developed a prognostic model based on two groups of patients, those with APAP-induced acute liver failure and those with non-APAP-induced acute liver failure. The first check is to obtain a blood gas measurement of arterial $\mathrm{pH}$; if arterial $\mathrm{pH}$ is $<7.3$ or blood lactate is $>3.0 \mathrm{mg} / \mathrm{dL}$ after adequate volume resuscitation, irrespective of level of encephalopathy, the patient meets the $\mathrm{KCH}$ Criteria. If acidosis or lactate does not meet the $\mathrm{KCH}$ criteria, another check is performed to determine whether the patient has a lactate $>3.5 \mathrm{mg} / \mathrm{dL}$ after early volume resuscitation. If this criteria is not met, the final set of criteria will be considered, which includes grade III or VI encephalopathy with prothrombin time $>100$ seconds or INR $>6.5$, with serum creatinine $>3.4 \mathrm{mg} / \mathrm{dL}$. In the absence of a liver transplant, the APAP-associated acute liver failure patients who met the $\mathrm{KCH}$ criteria reportedly have $80-90 \%$ mortality. ${ }^{51-53}$

Various other scoring systems have been evaluated as well. The model for end-stage liver disease (MELD) has not proven superior to the $\mathrm{KCH}$ criteria or INR alone. ${ }^{53}$ One study compared various scoring systems, including the $\mathrm{KCH}$ criteria, the sequential organ failure assessment (SOFA), the MELD and the acute physiology and chronic health evaluation II (APACHE II), as prognostic indicators for their ability to reduce mortality rates due to APAP hepatotoxicity. While the $\mathrm{KCH}$ criteria had excellent specificity, it was at the expense of sensitivity, yielding a limited ability to distinguish patients who would survive with conservative medical management versus those that would die without a liver transplant. ${ }^{52}$ The SOFA score, owing to its measures of physiologic parameters of multiple organ systems, was superior in discriminating patients who would benefit most from a liver transplant evaluation. ${ }^{52}$

Similar to any solid tumor transplant candidate, candidates for transplant treatment for APAP hepatotoxicity should undergo a thorough psychiatric evaluation and assessment to determine the level of social support needed. This type of evaluation becomes especially important in cases for which the APAP-associated liver failure was a consequence of intentional ingestion, as a repeat episode of over-ingestion will inevitably cause harm to the transplanted organ and squandering of a limited resource.

\section{Serum biomarkers of APAP-induced liver injury}

The detection of one or more biomarkers in the setting of APAP hepatotoxicity is desirable, especially in clinical scenarios where the diagnosis of APAP hepatotoxicity is unclear. Multiple serum biomarkers have been described as potential indicators, not only to identify hepatocyte injury and necrosis but also to help predict patient outcomes based on presence or absence of certain intracellular or intramitochondrial markers. ${ }^{12,17,63-69}$

APAP protein adducts that form upon NAPQI conjugation with protein sulfhydryl groups of cysteine in GSH or cellular proteins readily interact with mitochondria. These mitochondrial protein adducts are thought to cause mitochondrial dysfunction and to promote oxidant stress. ${ }^{17}$ Indeed, high levels of protein adducts have been detected in the serum of patients with APAP overdose. ${ }^{17}$ Detection of elevated levels of these APAP-protein adducts by high-pressure liquid chromatography (HPLC) can be used for clinical indication of APAP hepatotoxicity. However, such testing remains a specialized method, available to certain institutions, and thus has not spread to all health centers that handle cases of APAP hepatotoxicity. For HPLC interpretation of suspected APAP hepatotoxicity cases, liver centers will have to look to hire expert technicians who can provide these services in an emergency setting.

Serum markers of mitochondrial damage and death, including glutamate dehydrogenase (GDH), nuclear DNA (nDNA) and mtDNA have been investigated as clinically useful surrogate markers capable of indicating mitochondrial lysis following hepatocyte necrosis in APAP hepatotoxicity. ${ }^{12,64-68}$ There is evidence that patients who were non-survivors of APAPinduced acute liver failure had a statistically significant increase in GDH, nDNA, and mtDNA fragment levels, as compared to patients that recovered and survived their acute liver failure, inferring that more significant mitochondrial damage portends a higher mortality rate. ${ }^{65}$ The highest activity of $\mathrm{GDH}$ in plasma has been found in patients with markedly elevated ALT levels emanating from zone 3 of the liver, where $\mathrm{GDH}$ is most highly expressed and where APAP is known to case the greatest extent of liver injury. ${ }^{12}$ Moreover, the time course of the release of GDH and mtDNA has been shown to correspond well to the release of ALT from hepatocytes undergoing necrosis.

In addition to GDH, nDNA and mtDNA, the intranuclear product high mobility group B1 (HMGB1), a chromatin protein involved in nuclear DNA organization and transcription regulation, is also detectable in serum in the setting of hepatocyte necrosis. ${ }^{64}$ The detection of these serum biomarkers, also referred to as DAMPs, may help in the future decision-making of therapeutic designs and transplant evaluations, as well as to identify future potential targets of medical therapy. ${ }^{66}$

The association between a rise in levels of bile acids and those of ALT (indicating hepatocyte injury) was investigated as another biomarker that might be potentially useful for predicting prognosis of APAP hepatotoxicity. The process of bile acid transport from hepatocytes into the biliary tract via canalicular transporters is sensitive to disturbances, as evidenced by elevated bilirubin levels that occur upon minor liver injury. A research group found that one specific bile acid, glycodeoxycholic acid (GDCA), rose significantly higher in the group of non-surviving APAP-associated liver failure patients than in the group of patients who survived their liver failure despite having similar ALT levels as the first group. ${ }^{64}$ Due to the increased efficiency in medical diagnostics afforded by HPLC and mass spectroscopy techniques, prognostic-related findings of bile acid level may add to the armamentarium of findings from studies on how to better predict patient morbidity and mortality, and perhaps can even be used to shift treatment decision-making on surgical transplantation versus medical therapy for APAP overdose.

On the molecular level, human microRNAs (miRNA) have also been identified as potential early biomarkers for indicating APAP-induced hepatotoxicity at a stage before alanine aminotransferases become elevated. One miRNA in particular, the miRNA-122, was shown in a cohort who had taken APAP to significantly increase before ALT levels rose. ${ }^{67}$ This finding was reaffirmed in another study that demonstrated a 
corresponding increase of serum miRNA-122 and miRNA-375, along with an increase in APAP protein adducts, all of which were also seen prior to ALT elevation. ${ }^{68}$ Urinary miRNA-375 was also detected at high levels in that study. ${ }^{68}$ Furthermore, that study had also enrolled children who had presented to the hospital with accidental APAP overdose, and thus applicability to the adult population may be better reserved for a time after other studies are completed with a greater adult population.

One research group has sought to capture one aspect of the wide systemic effects of APAP hepatotoxicity by focusing in on kidney failure following acute APAP ingestion. Specifically, expression of the kidney injury molecule-1 (KIM-1) was quantified, and it was found that patients who died from APAP-associated acute liver failure or who required liver transplant had significantly higher KIM-1 values on day 1 of presentation, as compared to those who spontaneously survived. ${ }^{69}$ Elevations of a kidney injury marker should not come as a surprise, however, as acute kidney injury evaluation in the form of serum creatinine is included in scoring systems such as the APACHE II and $\mathrm{KCH}$ criteria. KIM-1 measurements appear to outperform traditional serum creatinine as a measure of acute organ damage, and this possibility deserves further research attention.

Detection of biomarkers is certain to gain more clinical significance in the future management of APAP hepatotoxicity in the modern era of outcome-based medical care. Collective qualitative or quantitative results of these biomarkers may serve to predict potential morbidity and/or mortality of liver disease and failure, leading to appropriate triage of patients into those requiring emergency liver transplantation versus patients who are likely to have good clinical improvement with NAC therapy alone. More validated studies need to be done to make this correlation apparent.

\section{In the hospital: acute management of APAP overdose}

Physically blocking APAP from the GI tract: gastrointestinal decontamination

One proposed method of initial therapy is to physically inhibit drug absorption or remove the offending agent from the gastrointestinal tract. If a patient is fortunate enough to seek out and obtain early medical attention (typically within 4 hours of the acute APAP ingestion), activated charcoal can be effective in limiting drug absorption, unless there are obvious contraindications, such as an unprotected airway or compromise of the gastrointestinal tract. ${ }^{70}$ Gastric lavage, activated charcoal ingestion, and vomiting induced by ipececauanha can all reduce drug absorption, but weak evidence suggests activated charcoal as the preferred method of gastrointestinal decontamination. ${ }^{71}$ The only patients who may benefit from activated charcoal beyond 4 hours of ingestion are those who ingested extended-release APAP formulations or co-ingestion of drugs that delay gastric emptying time. ${ }^{70}$ Use of activated charcoal is not without risks, however, and one must be wary of aspiration pneumonia, vomiting, diarrhea, constipation, ileus, and interference with the patient's regular medications. ${ }^{72}$

\section{NAC}

NAC is a cysteine prodrug and hepatic GSH precursor, and should be administered immediately as an antidote in patients with established APAP hepatotoxicity or those with high risk of developing this condition. NAC replenishes and maintains hepatic GSH stores by providing cysteine, the substrate which detoxifies reactive metabolites of APAP. ${ }^{23,24,56,75}$ In addition, it may reduce NAPQI back to APAP by enhancing the sulfonation pathway of APAP metabolism. ${ }^{56,75}$ Administering NAC to patients with APAP overdose may reduce mortality from $5 \%$ to $0.7 \%$ of patients, and thus timely administration is critical. ${ }^{71}$ Table 2 lists commonly accepted indications for treatment with NAC in the setting of acute over-ingestion.

Delaying therapy to APAP hepatotoxic patients is dangerous. While the goal is to treat patients before the development of acute liver failure, treatment should still be given to patients who are found to be in active hepatic failure since NAC appears to improve hepatic perfusion and oxygen delivery, to refine mitochondrial energy metabolism, and to facilitate scavenging of reactive oxygen and nitrogen species. ${ }^{56,60-62}$ NAC can be delivered as either intravenous (IV) or oral (PO) preparations, with dosing based on the patient's body weight (maximum dose of $110 \mathrm{~kg}$ for oral and $100 \mathrm{~kg}$ for IV). ${ }^{54,56,71}$

The standard formulations for NAC are as follows. For the IV regimen, a loading dose of $150 \mathrm{mg} / \mathrm{kg}$ in $200 \mathrm{cc}$ is given for 15 minutes, followed by $50 \mathrm{mg} / \mathrm{kg}$ in $500 \mathrm{cc}$ over 4 hours and then $100 \mathrm{mg} / \mathrm{kg}$ in $1000 \mathrm{cc}$ over 16 hours. ${ }^{4,54,56,71}$ For the PO regimen, a loading dose of $140 \mathrm{mg} / \mathrm{kg}$ is given, followed by $70 \mathrm{mg} / \mathrm{kg}$ every 4 hours for a total of 18 total doses. ${ }^{54,56,71}$ Renal or hepatic dose adjustment is unnecessary. Patients at high risk of hepatotoxicity should have serial APAP level and hepatic function panels measured before the completion of NAC. NAC should be continued if APAP levels remain $>10 \mu \mathrm{g} / \mathrm{mL}$ or ALT is persistently elevated, with reevaluation of the status after 12 hours. ${ }^{54,56}$ NAC therapy can be considered complete if APAP levels are undetectable and ALT level has returned to normal. ${ }^{56}$ However, if the patient continues to present evidence of liver dysfunction beyond persistent ALT elevation (satisfying $\mathrm{KCH}$ criteria of acidosis, elevated creatinine, hyperbilirubiniemia, coagulopathy and encephalopathy), then NAC therapy should be continued as

\section{Table 2. Clinical indications for use of NAC}

\begin{tabular}{|c|c|}
\hline 1 & Severe APAP toxicity with ALT:AST > 1000 \\
\hline 2 & Initiation of NAC within 24 hours of ingestion \\
\hline 3 & $\begin{array}{l}\text { Serum APAP levels from } 140 \mathrm{mg} / \mathrm{L} \text { at } 4 \text { hours to } \\
50 \mathrm{mg} / \mathrm{L} \text { at } 10 \text { hours }\end{array}$ \\
\hline 4 & $\begin{array}{l}\text { Acute poisoning (ingested in } 1 \text { hour) with no other } \\
\text { products containing acetaminophen in the past } 24 \\
\text { hours }\end{array}$ \\
\hline 5 & $\begin{array}{l}\text { Acute poisoning with no ingestion of sustained release } \\
\text { formulations }\end{array}$ \\
\hline 6 & Baseline normal ALT, AST and INR \\
\hline 7 & $\begin{array}{l}\text { Used ideally within the first } 8-10 \text { hours with risk of } \\
\text { hepatotoxicity being }<5 \% \text {, especially if APAP level is } \\
\text { above the treatment line on the Rumack-Matthew } \\
\text { nomogram }\end{array}$ \\
\hline 8 & $\begin{array}{l}\text { Empirical use when APAP levels cannot be obtained } \\
\text { within } 8 \text { hours of ingestion }\end{array}$ \\
\hline
\end{tabular}

Abbreviations: APAP, acetaminophen; ALT, alanine aminotransferase; AST, aspartate aminotransferase; INR, international normalized ratio; NAC, $\mathrm{N}$-acetylcysteine. 
these indicators portend a poor prognosis regardless of transaminase levels. ${ }^{62}$ Patients with severe hepatotoxicity or acute liver failure should continue on NAC at $6.25 \mathrm{mg} / \mathrm{kg} / \mathrm{h}$ until a liver transplant takes place or liver dysfunction is reversed (evidenced by regression of AST or ALT, resolved encephalopathy, and/or INR > 1.5) with an undetectable APAP level. ${ }^{54,56}$ Table 3 describes how NAC therapy functions to mitigate hepatocyte necrosis and to promote liver regeneration.

While there are two treatment options, the clinical scenario should determine the route of NAC administration (PO versus IV). The efficacy between the two preparations appears the same, although no head-to-head trial currently exists. IV NAC is preferentially given to patients with acute liver failure and to those who refuse or have a contraindication for the PO formulation; contraindications include coma, pancreatitis, ileus or gastrointestinal tract insufficiency. IV NAC has a $10-20 \%$ risk of anaphylactic reaction, and transfusion should not be halted for minor reactions of flushing. ${ }^{60,72}$ Other identified side-effects of NAC include nausea, vomiting, diarrhea or constipation, and rarely fever, headache, drowsiness or hypotension. Steroids, diphenhydramine and bronchodilators can help resolve the anaphylactoid reactions and should be given so that NAC therapy can be continued under close monitoring. ${ }^{72}$ The oral formulation has a sulfur component, and thus has an unpleasant taste and smell that can cause nausea and vomiting; switching PO to IV therapy is an option. This switch can be mitigated, however, by using a nasogastric tube for direct gastrointestinal access or by taking the medication with sodas or juice to make the medication more tolerable.

\section{Liver transplantation}

Liver transplantation can be a lifesaving procedure when APAP ingestion has progressed to irreversible liver failure. Prognostic factors including MELD, KCH criteria and APACHE II scores have all been investigated as objective indicators to select liver transplantation candidates. ${ }^{51,52,62}$ Qualifying for liver transplantation not only requires a projected poor outcome and high risk of mortality based on these scoring

Table 3. Role of NAC

1 Protection against reactive oxygen species by increasing Nrf2 and HO-1 mRNA levels

2 Protection against mitochondrial dysfunction, which causes release of acylcarnitines in peripheral vasculature

3 Elimination of JNK activation and GDH release

4 The mechanism of protection during the early metabolism phase primarily involves improved scavenging of the reactive metabolite NAPQI due to accelerated GSH synthesis

5 Scavenging of the reactive metabolite NAPQI and decreasing of protein binding during the early phase occurs through increasing levels of GSH

6 Provision of increased amounts of cysteine to allow regeneration of GSH well beyond clearance of APAP, allowing for hepatocyte regeneration

Abbreviations: JNK, c-Jun-N-terminal kinase; GD, glutamate dehydrogenase; $\mathrm{GSH}$, glutathione; NAC, $\mathrm{N}$-acetylcysteine; HO-1, heme oxygenase-1; NAPQI $\mathrm{N}$-acetyl-para-benzo-quinone imine; Nif2, nuclear factor erythroid 2-related factor 2. systems but also necessitates a multidisciplinary approach that includes psychiatric evaluation, which may be challenging for patients who have intentionally overdosed on APAP. In one study, $20 \%$ of the center's liver transplantation cases were due to APAP overdose, which was the most common cause of acute liver failure prompting transplantation; however, only $12 \%$ of those patients presenting to this same center with acute liver failure from APAP overdose actually received a transplant. ${ }^{51}$ In a large multicenter study of liver failure cases from the United States' Acute Liver Failure Study Group, $54 \%$ of patients had APAP-related liver failure and were compared to non-APAP related liver failure patients; the APAP-associated liver failure cases fared better in 2-year survivorship if they were not transplanted, as compared to transplanted patients. ${ }^{73}$ Conservative medical therapy has a good outcome when prognostic scoring systems point against proceeding with transplant, and it appears that physicians are making better-informed decisions in regards to transplantation of patients who present with acute liver failure related to APAP hepatotoxicity.

\section{Alternative therapeutic thoughts in APAP hepatotoxicity}

Targeting APAP hepatotoxicity at the molecular level as an alternative to NAC therapy has been the goal of many research groups. One particular group has been investigating metformin as a potential therapeutic intervention to reduce hepatotoxicity after APAP exposure. The proposed therapeutic mechanism involves the down-regulation of c-Jun-N-terminal kinase (JNK) by inducing Gadd45-beta activity, which ultimately disrupts a mechanism of mitochondrial damage. ${ }^{74}$

One investigative group in South Korea has reported on the use of sulforaphane (SFN) as a potential protector against oxidant-induced tissue injury. ${ }^{75}$ SFN is a dietary isothiocynanate synthesized from a precursor found in vegetables such as cauliflower, broccoli, kale, cabbage, collards and Brussels sprouts as well as other genera, such as the radish. ${ }^{75}$ SFN has been previously shown to have protective effects against oxidative stress-damaged tissues, such as the brain, heart, pancreas and kidney. ${ }^{75}$ In primary hepatocytes, cells pretreated with SFN were shown to be protected against APAPinduced liver injuries via antioxidant actions by blocking generation of reactive oxygen species, depletion of GSH and peroxidation of lipids. ${ }^{75}$

Another group in China has suggested an association between adequate hepatocellular serotonin levels in murine models and protection against hepatocyte inflammation, oxidative stress, GSH depletion, peroxynitrite formation, elevated hepatocyte proliferation and reduction in several enzymes described in the process of hepatocyte necrosis. ${ }^{76}$ Human hepatocyte models are a next logical step, and thus more investigations need to be carried out before either serotonin or SFN can be realistically considered on the therapeutic level.

\section{Conclusion}

APAP ingestion and subsequent hepatotoxicity is a critical problem that continues to plague individuals across the world, due to the cheap cost of APAP contributing to its being a ubiquitous analgesic and anti-pyretic drug available through consumer pharmacies and as prescription-only medication formulations. Since APAP is responsible for nearly half of the cases of acute liver failure in the United States and remains 
the leading cause for liver transplantation, continued awareness, education and research should be undertaken. ${ }^{78}$ Perhaps due to the attention paid to APAP-induced acute liver failure, survival rates of $\sim 60 \%$ are touted as decent when compared to DILI from other substances. ${ }^{77}$ New research in detecting biomarkers of injured and necrotic hepatocytes seems promising, especially since it has become increasingly important to identify APAP-induced acute liver injury patients earlier in order to provide lifesaving medical and surgical therapies. While much is currently known about APAP hepatotoxicity regarding its epidemiology, risk factors, pharmacology and toxicology, diagnostics and treatment modalities, there remains a plethora of scientific questions that should be answered in order to improve the understanding of molecular and sub-molecular relationships and pathways that may offer new therapeutics to tackle this curable yet potentially devastating event.

\section{Conflict of interest}

None

\section{Author contributions}

Contributed to the manuscript by drafting the document, formatting the figures and tables, obtaining permission for use of previously published figures, and performing final editing of the content (EY), contributed to the manuscript by writing content and formatting the tables in the manuscript $(A B)$, contributed to the manuscript by drafting sections of the document $(M C)$, contributed to the manuscript by providing editing comments (MK), contributed to the manuscript by providing critical revision and administration during the manuscript writing (NP).

\section{References}

[1] Bunchorntavakul C, Reddy KR. Acetaminophen-related Hepatotoxicity. Clin Liver Dis 2013;17:587-607. doi: 10.1016/j.cld.2013.07.005.

[2] Herndon CM, Dankenbring DM. Patient perception and knowledge of acetaminophen in a large family medicine service. J Pain Palliat Care Pharmacother 2014;28:109-116. doi: 10.3109/15360288.2014.908993.

[3] Clark R, Fisher JE, Sketris IS, Johnston GM. Population prevalence of high dose paracetamol in dispensed paracetamol/opioid prescription combinations: an observational study. BMC Clin Pharmacol 2012;12:11. doi: 10. 1186/1472-6904-12-11.

[4] Murray KF, Hadzic N, Wirth S, Bassett M, Kelly D. Drug-related hepatotoxicity and acute liver failure. J Pediatr Gastroenterol Nutr 2008;47:395-405. doi: 10.1097/MPG.0b013e3181709464.

[5] Michna E, Duh MS, Korves C, Dahl JL. Removal of opioid/acetaminophen combination prescription pain medications: assessing the evidence for hepatotoxicity and consequences of removal of these medications. Pain Med 2010;11:369-378. doi: 10.1111/j.1526-4637.2010.00811.x.

[6] Blieden M, Paramore LC, Shah D, Ben-Joseph R. A perspective on the epidemiology of acetaminophen exposure and toxicity in the United States. Expert Rev Clin Pharmacol 2014;7:341-348. doi: 10.1586/17512433. 2014.904744.

[7] Hughes B, Durran A, Langford NJ, Mutimer D. Paracetamol poisoning impact of pack size restrictions. J Clin Pharm Ther 2003;28:307-310. doi: 10.1046/j.1365-2710.2003.00497.x.

[8] McGill MR, Jaeschke H. Metabolism and disposition of acetaminophen: recent advances in relation to hepatotoxicity and diagnosis. Pharm Res 2013;30: 2174-2187. doi: 10.1007/s11095-013-1007-6.

[9] Jaeschke H, McGill MR, Ramachandran A. Oxidant stress, mitochondria, and cell death mechanisms in drug-induced liver injury: lessons learned from acetaminophen hepatotoxicity. Drug Metab Rev 2012;44:88-106. doi: 10. 3109/03602532.2011.602688.

[10] Jaeschke H, Williams CD, Ramachandran A, Bajt ML. Acetaminophen hepatotoxicity and repair: the role of sterile inflammation and innate immunity. Liver Int 2012;32:8-20. doi: 10.1111/j.1478-3231.2011.02501.x.
[11] Yuan L, Kaplowitz N. Mechanisms of drug-induced liver injury. Clin Liver Dis 2013;17:507-518. doi: 10.1016/j.cld.2013.07.002.

[12] McGill MR, Sharpe MR, Williams CD, Taha M, Curry SC, Jaeschke H. The mechanism underlying acetaminophen-induced hepatotoxicity in humans and mice involves mitochondrial damage and nuclear DNA fragmentation. J Clin Invest 2012;122:1574-1583. doi: 10.1172/JCI59755.

[13] Jaeschke H, McGill MR. Cytochrome P450-derived versus mitochondrial oxidant stress in acetaminophen hepatotoxicity. Toxicol Lett 2015;235: 216-217. doi: 10.1016/j.toxlet.2015.04.002.

[14] Xie Y, Ramachandran A, Breckenridge DG, Liles JT, Lebofsky M, Farhood A, et al. Inhibitor of apoptosis signal-regulating kinase 1 protects against acetaminophen-induced liver injury. Toxicol Appl Pharmacol 2015;286:1-9. doi: 10. 1016/j.taap.2015.03.019.

[15] Liu ZX, Govindarajan S, Kaplowitz N. Innate immune system plays a critical role in determining the progression and severity of acetaminophen hepatotoxicity. Gastroenterology 2004;127:1760-1774. doi: 10.1053/j.gastro. 2004.08.053.

[16] Jaeschke $H$. Role of inflammation in the mechanism of acetaminopheninduced hepatotoxicity. Expert Opin Drug Metab Toxicol 2005;1:389-397. doi: $10.1517 / 17425255.1 .3 .389$.

[17] Xie Y, McGill MR, Dorko K, Kumer SC, Schmitt TM, Forster J, et al. Mechanisms of acetaminophen-induced cell death in primary human hepatocytes. Toxicol Appl Pharmacol 2014;279:266-274. doi: 10.1016/j.taap.2014.05. 010 .

[18] Douglas DR, Sholar JB, Smilkstein MJ. A pharmacokinetic comparison of acetaminophen products (Tylenol Extended Relief vs regular Tylenol). Acad Emerg Med 1996;3:740-744. doi: 10.1111/j.1553-2712.1996.tb03508.x.

[19] Watkins PB, Kaplowitz N, Slattery JT, Colonese CR, Colucci SV, Stewart PW, et al. Aminotransferase elevations in healthy adults receiving 4 grams of acetaminophen daily: a randomized controlled trial. JAMA 2006;296:87-93. doi: $10.1001 /$ jama.296.1.87.

[20] Schmidt LE, Dalhoff K, Poulsen HE. Acute versus chronic alcohol consumption in acetaminophen-induced hepatotoxicity. Hepatology 2002;35:876882. doi: $10.1053 /$ jhep.2002.32148.

[21] Dart RC, Erdman AR, Olson KR, Christianson G, Manoguerra AS, Chyka PA, et al. Acetaminophen poisoning: an evidence-based consensus guideline for out-of-hospital management. Clin Toxicol (Phila) 2006;44:1-18. doi: 10.1080/15563650500394571.

[22] Waring WS, Stephen AF, Malkowska AM, Robinson OD. Acute ethanol coingestion confers a lower risk of hepatotoxicity after deliberate acetaminophen overdose. Acad Emerg Med 2008;15:54-58. doi: 10.1111/j.1553-2712. 2007.00019.x.

[23] Zhao P, Slattery JT. Effects of ethanol dose and ethanol withdrawal on rat liver mitochondrial glutathione: implication of potentiated acetaminophen toxicity in alcoholics. Drug Metab Dispos 2002;30:1413-1417. doi: 10. 1124/dmd.30.12.1413

[24] Lauterburg BH, Velez ME. Glutathione deficiency in alcoholics: risk factor for paracetamol hepatotoxicity. Gut 1988;29:1153-1157. doi: 10.1136/gut.29. 9.1153.

[25] Abebe W. Herbal medication: potential for adverse interactions with analgesic drugs. J Clin Pharm Ther 2002;27:391-401. doi: 10.1046/j.1365-2710. 2002.00444.x

[26] Marzilawati AR, Ngau YY, Mahadeva S. Low rates of hepatotoxicity among Asian patients with paracetamol overdose: a review of 1024 cases. BMC Pharmacol Toxicol 2012;13:8. doi: 10.1186/2050-6511-13-8

[27] Rumack BH. Acetaminophen misconceptions. Hepatology 2004;40:10-15. doi: $10.1002 /$ hep.20300

[28] Mennecozzi M, Landesmann B, Palosaari T, Harris G, Whelan M. Sex differences in liver toxicity-do female and male human primary hepatocytes react differently to toxicants in vitro? PLoS One 2015;10:e0122786. doi: 10. 1371/journal.pone.0122786.

[29] Zenger F, Russmann S, Junker E, Wuthrich C, Bui MH, Lauterburg BH. Decreased glutathione in patients with anorexia nervosa. Risk factor for toxic liver injury? Eur J Clin Nutr 2004;58:238-243. doi: 10.1038/sj.ejcn. 1601772.

[30] Whitcomb DC, Block GD. Association of acetaminophen hepatotoxicity with fasting and ethanol use. JAMA 1994;272:1845-1850. doi: 10.1001/jama. 1994.03520230055038.

[31] Organ-specific warnings: internal analgesic, antipyretic, and antirheumatic drug products for over-the-counter human use-labeling for products that contain acetaminophen; guidance for industry; availability. US Department of Health and Human Services Food and Drug Administration Center for Drug Evaluation and Research (CDER) 2015.

[32] Krenzelok EP, Royal MA. Confusion: acetaminophen dosing changes based on NO evidence in adults. Drugs R D 2012;12:45-48. doi: $10.2165 / 11633010-$ 000000000-00000.

[33] Jaeschke H, Williams CD, McGill MR, Farhood A. Herbal extracts as hepatoprotectants against acetaminophen hepatotoxicity. World J Gastroenterol 2010;16:2448-2450. doi: 10.3748/wjg.v16.i19.2448. 
[34] Jiang $Y$, Fan $X$, Wang $Y$, Chen $P$, Zeng $H$, Tan $H$, et al. Schisandrol $B$ protects against acetaminophen-induced hepatotoxicity by inhibition of CYP-mediated bioactivation and regulation of liver regeneration. Toxicol Sci $2015 ; 143$ : 107-115. doi: 10.1093/toxsci/kfu216.

[35] Dawson DJ, Babbs C, Warnes TW, Neary RH. Hypophosphatemia in acute liver failure. Br Med J (Clin Res Ed) 1987;295:1312-1313. doi: 10.1136/ bmj.295.6609.1312.

[36] Fontana RJ, Ellerbe C, Durkalski VE, Rangnekar A, Reddy RK, Stravitz T, et al. Two-year outcomes in initial survivors with acute liver failure: results from a prospective, multicentre study. Liver Int 2015;35:370-380. doi: 10. 1111 /liv. 12632.

[37] Schmidt LE, Dalhoff K. Serum phosphate is an early predictor of outcome in severe acetaminophen-induced hepatotoxicity. Hepatology 2002;36: 659-665. doi: 10.1053/jhep.2002.35069.

[38] Ng KL, Davidson JS, Bathgate AJ. Serum phosphate is not a reliable early predictor of outcome in paracetamol induced hepatotoxicity. Liver Transpl 2004;10:158-159. doi: 10.1002/It.20022.

[39] Wang DW, Yin YM, Yao YM. Advances in the management of acute liver failure. World J Gastroenterol 2013;19:7069-7077. doi: 10.3748/wjg.v19.i41.7069.

[40] Possamai LA, McPhail MJ, Quaglia A, Zingarelli V, Abeles RD, Tidswell R, et al. Character and temporal evolution of apoptosis in acetaminophen-induced acute liver failure. Crit Care Med 2013;41:2543-2550. doi: 10.1097/CCM. 0b013e31829791a2.

[41] Bari K, Fontana RJ. Acetaminophen overdose: what practitioners need to know. Clinical Liver Dis 2014;4:17-21. doi: 10.1002/cld.373.

[42] Rumore MM, Blaiklock RG. Influence of age-dependent pharmacokinetics and metabolism on acetaminophen hepatotoxicity. J Pharm Sci 1992;81: 203-207. doi: 10.1002/jps.2600810302.

[43] Critchley JA, Nimmo GR, Gregson CA, Woolhouse NM, Prescott LF. Intersubject and ethnic differences in paracetamol metabolism. Br J Clin Pharmacol 1986;22:649-657. doi: 10.1111/j.1365-2125.1986.tb02953.x.

[44] Ueshima Y, Tsutsumi M, Takase S, Matsuda Y, Kawahara H. Acetaminophen metabolism in patients with different cytochrome P-4502E1 genotypes. Alcohol Clin Exp Res 1996;20:25A-28A. doi: 10.1111/j.1530-0277.1996. tb01722.x.

[45] Zhao L, Pickering G. Paracetamol metabolism and related genetic differences. Drug Metab Rev 2011;43:41-52. doi: 10.3109/03602532.2010.527984.

[46] Wilkes JM, Clark LE, Herrera JL. Acetaminophen overdose in pregnancy. South Med J 2005;98:1118-1122. doi: 10.1097/01.smj.0000184792.15407.51.

[47] Thornton SL, Minns AB. Unintentional chronic acetaminophen poisoning during pregnancy resulting in liver transplantation. J Med Toxicol 2012;8: 176-178. doi: 10.1007/s13181-012-0218-2.

[48] Mazer M, Perrone J. Acetaminophen-inducted nephrotoxicity: pathophysiology, clinical manifestations, and management. J Med Toxicol 2008;4:2-6. doi: $10.1007 / B F 03160941$.

[49] Caldarola V, Hassett JM, Hall AH, Bronstein AB, Kulig KW, Rumack BH. Hemorrhagic pancreatitis associated with acetaminophen overdose. Am J Gastroenterol 1986;81:579-582.

[50] Harrison PM, O'Grady JG, Keays RT, Alexander GJ, Williams R. Serial prothrombin time as prognostic indicator in paracetamol induced fulminant hepatic failure. BM] 1990;301:964-966. doi: 10.1136/bmj.301.6758.964.

[51] Bailey B, Amre DK, Gaudreault P. Fulminant hepatic failure secondary to acetaminophen poisoning: a systematic review and meta-analysis of prognostic criteria determining the need for liver transplantation. Crit Care Med 2003;31:299-305. doi: 10.1097/00003246-200301000-00048.

[52] Cholongitas E, Theocharidou E, Vasianopoulou P, Betrosian A, Shaw S, Patch $D$, et al. Comparison of the sequential organ failure assessment score with the King's College Hospital criteria and the model for end-stage liver disease score for the prognosis of acetaminophen-induced acute liver failure. Liver Transpl 2012;18:405-412. doi: 10.1002/It.23370.

[53] Craig DG, Ford AC, Hayes PC, Simpson KJ. Systematic review: prognostic tests of paracetamol-induced acute liver failure. Aliment Pharmacol Ther 2010;31:1064-1076. doi: 10.1111/j.1365-2036.2010.04279.x.

[54] Rumack BH. Acetaminiphen hepatotoxicity: the first 35 years. J Toxicol Clin Toxicol 2002;40:3-20. doi: 10.1081/CLT-120002882.

[55] Buehler MC, Curry SC. False positive acetaminophen levels associated with hyperbilirubinemia. Clin Toxicol (Phila) 2005;43:167-170. doi: 10.1081/ CLT-200057853.

[56] Heard KJ. Acetylcysteine for acetaminophen poisoning. N Engl J Med 2008; 359:285-292. doi: 10.1056/NEJMct0708278.

[57] Prescott LF, Illingworth RN, Critchley JA, Stewart MJ, Adam RD, Proudfoot AT. Intravenous $\mathrm{N}$-acetylcysteine: the treatment of choice for paracetamol poisoning. Br Med J 1979;2:1097-1100. doi: 10.1136/bmj.2.6198.1097.
[58] Harrison PM, Wendon JA, Gimson AE, Alexander GJ, Williams R. Improvement by acetylcysteine of hemodynamics and oxygen transport in fulminant hepatic failure. N Engl J Med 1991;324:1852-1857. doi: 10.1056/ NEJM199106273242604.

[59] Smilkstein MJ, Knapp GL, Kulig KW, Rumack BH. Efficiency of oral $\mathrm{N}$-acetylcysteine in the treatment of acetaminophen overdose. Analysis of the national multicenter study (1976 to 1985). N Engl J Med 1988;319: 1557-1562. doi: 10.1056/NEJM198812153192401.

[60] Kerr F, Dawson A, Whyte IM, Buckley N, Murray L, Graudins A, et al. The Australasian Clinical Toxicology Investigators Collaboration randomized trial of different loading infusion rates of $\mathrm{N}$-acetylcysteine. Ann Emerg Med 2005;45:402-408. doi: 10.1016/j.annemergmed.2004.08.040.

[61] de Andrade KQ, Moura FA, dos Santos JM, de Araújo OR, de Farias Santos JC, Goulart MO. Oxidative stress and inflammation in hepatic diseases: therapeutic possibilities of $\mathrm{N}$-acetylcysteine. Int J Mol Sci 2015;16:30269-30308. doi: $10.3390 /$ ijms 161226225 .

[62] McGovern AJ, Vitkovitsky IV, Jones DL, Mullins ME. Can AST/ALT ratio indicate recovery after acute paracetamol poisoning? Clin Toxicol (Phila) 2015;53: 164-167. doi: 10.3109/15563650.2015.1006399.

[63] Xie Y, McGill MR, Cook SF, Sharpe MR, Winefield RD, Wilkins DG, et al. Time course of acetaminophen-protein adducts and acetaminophen metabolites in circulation of overdose patients and in HepaRG cells. Xenobiotica 2015;45: 921-929. doi: 10.3109/00498254.2015.1026426.

[64] Woolbright BL, McGill MR, Staggs VS, Winefield RD, Gholami P, Olyaee M, et al. Glycodeoxycholic acid levels as prognostic biomarker in acetaminopheninduced acute liver failure patients. Toxicol Sci 2014;142:436-444. doi: 10. 1093/toxsci/kfu195

[65] McGill MR, Staggs VS, Sharpe MR, Lee WM, Jaeschke H, Acute Liver Failure Study Group. Serum mitochondrial biomarkers and damage-associated molecular patterns are higher in acetaminophen overdose patients with poor outcome. Hepatology 2014;60:1336-1345. doi: 10.1002/hep.27265.

[66] McGill MR, Jaeschke H. Mechanistic biomarkers in acetaminophen-induced hepatotoxicity and acute liver failure: from preclinical models to patients. Expert Opin Drug Metab Toxicol 2014;10:1005-1017. doi: 10.1517/ 17425255.2014 .920823

[67] Thulin P, Nordahl G, Gry M, Yimer G, Aklillu E, Makonnen E, et al. Keratin-18 and microRNA-122 compliment alanine aminotransferase as novel safety biomarkers for drug-induced liver injury in two human cohorts. Liver Int 2014;34:367-378. doi: 10.1111/liv.12322.

[68] Yang X, Salminen WF, Shi Q, Greenhaw J, Gill PS, Bhattacharyya S, et al. Potential of extracellular microRNAs as biomarkers of acetaminophen toxicity in children. Toxicol Appl Pharmacol 2015;284:180-187. doi: 10.1016/j.taap. 2015.02.013.

[69] Antoine DJ, Sabbisetti VS, Francis B, Jorgensen AL, Craig DG, Simpson KJ, et al. Circulating kidney injury molecule 1 predicts prognosis and poor outcome in patients with acetaminophen-induced liver injury. Hepatology 2015;62:591-599. doi: 10.1002/hep.27857.

[70] Underhill TJ, Greene MK, Dove AF. A comparison of the efficacy of gastric lavage, ipecacuanha and activated charcoal in the emergency management of paracetamol overdose. Arch Emerg Med 1990;7:148-154. doi: 10.1136/ emj.7.3.148.

[71] Chun LJ, Tong MJ, Busuttil RW, Hiatt JR. Acetaminophen hepatotoxicity and acute liver failure. J Clin Gastroenterol 2009;43:342-349. doi: 10.1097/ MCG.0b013e31818a3854

[72] Buckley N, Eddleston M. Paracetamol (acetaminophen) poisoning. BM] Clin Evid 2007;2007.

[73] Reddy KR, Schilsky ML, Stravitz R, Ellerbe C, Durkalski V, Fontana RJ, et al. Liver transplantation for acute liver failure: results from the NIH Acute Liver Failure Study Group. Hepatology 2012;56:246A-247A.

[74] Kaplowitz N, Win S, Than TA, Liu ZX, Dara L. Targeting signal transduction pathways which regulate necrosis in acetaminophen hepatotoxicity. J Hepatol 2015;63:5-7. doi: 10.1016/j.jhep.2015.02.050.

[75] Noh JR, Kim YH, Hwang JH, Choi DH, Kim KS, Oh WK, et al. Sulforaphane protects against acetaminophen-induced hepatotoxicity. Food Chem Toxicol 2015;80:193-200. doi: 10.1016/j.fct.2015.03.020

[76] Zhang J, Song S, Pang Q, Zhang R, Zhou L, Liu S, et al. Serotonin deficiency exacerbates acetaminophen-induced liver toxicity in mice. Sci Rep 2015;5: 8098. doi: 10.1038/srep08098.

[77] Bliebel W, Kim S, D'Silva K, Lemmer ER. Drug-induced liver injury: review article. Dig Dis Sci 2007;52:2463-2471. doi: 10.1007/s10620-006-9472-y. 